[Communication]

\title{
Parasitos em trutas-arco-íris (Oncorhynchus mykiss) criadas em sistema intensivo na região serrana de Santa Catarina, Brasil
}

\author{
[Parasites in rainbow trout (Oncorhynchus mykiss) raised in an intensive system \\ in the mountain region of Santa Catarina, Brazil]
}

\author{
S.M. Henrique, G.V.T. Pilati, A. Snak, B.B. Costa, \\ A.R. Aguiar, E. Skoronski, A.B. Moura
}

Universidade do Estado de Santa Catarina - Lages, SC

\begin{abstract}
Doenças parasitárias em peixes de cultivo causam problemas, principalmente em altas densidades, limitando o rendimento da produção. Mesmo em infecções baixas, os parasitos podem propiciar a proliferação de bactérias e fungos em seus hospedeiros, resultando em infecções secundárias que podem levar o hospedeiro à morte (Marengoni et al., 2009).
\end{abstract}

Oncorhynchus mykiss é um Salmonidae, anádromo, encontrado naturalmente desde o Alaska até o México. O sistema mais comum para sua produção é o intensivo, com temperatura ideal de água entre 12 e $21^{\circ} \mathrm{C}$ e límpida, pois essa espécie é exigente quanto ao ambiente onde vive. O nível de oxigênio dissolvido exigido é de $9 \mathrm{mg} / \mathrm{L}$ de água (FAO, 2019).

Este trabalho teve como objetivo determinar a prevalência e a intensidade média de parasitos em O. mykiss, criados em sistema intensivo, na região serrana de Santa Catarina, e avaliar se há relação entre os parasitos observados com a qualidade da água dos viveiros.

Este projeto foi aprovado pela Comissão de Ética no Uso de Animais (Ceua) da Universidade do Estado de Santa Catarina (Udesc), sob protocolo $\mathrm{n}^{\circ}$ 5148190318. O estudo foi desenvolvido com trutas-arco-íris obtidas na linha de inspeção de uma indústria de pescado da região serrana de Santa Catarina, no município de Lages. Ao todo foram analisados 120 peixes, coletados entre os meses de abril a setembro de 2018 (abrangendo, respectivamente, outono e inverno).
Após a biometria (comprimento padrão e massa corpórea) e necropsia, foi realizada a coleta de material (muco, arcos branquiais, intestino, fígado e rim), conforme Jerônimo et al. (2012a), que foram acondicionados em formol tamponado $10 \%$ e encaminhados ao Laboratório de Parasitologia e Doenças Parasitárias do CAV/Udesc, para a pesquisa e identificação dos parasitos, seguindo Jerônimo et al. (2012b); Martins; Guiraldelli (2008). A cavidade visceral foi analisada no local da coleta, por meio de inspeção visual, buscando encontrar pontos hemorrágicos, cistos ou larvas; a musculatura foi analisada por meio do "candling table", sendo possível visualizar larvas encistadas no tecido.

Foi avaliada a qualidade da água das três propriedades produtoras dos peixes. As amostras de água $(1000 \mathrm{~mL})$, coletadas pela técnica de submersão, $15 \mathrm{~cm}$ abaixo da superfície de água, em recipiente de vidro estéril, foram mantidas refrigeradas $\left(10^{\circ} \mathrm{C}\right)$ e encaminhadas ao Laboratório de Tratamento de Águas e Resíduos (Labtrat), do Departamento de Engenharia Ambiental e Sanitária do CAV/Udesc. Foram analisados a concentração de amônia e de oxigênio dissolvido, o $\mathrm{pH}$, a temperatura e a turbidez. A prevalência e a intensidade média de parasitos foram obtidas de acordo com Bush et al. (1997). A relação entre a prevalência, a intensidade média, e quantidade de parasitos encontrados e qualidade da água foram obtidos por meio do teste de coeficiente de Pearson, com intervalo de confiança de $99 \%$, utilizando o programa BioEstat 5.0 (2018).

Recebido em 19 de agosto de 2019

Aceito em 25 de maio de 2020

E-mail: schayanne.henrique@gmail.com 
Todos os espécimes analisados possuíam comprimento padrão variando entre 23 e $34 \mathrm{~cm}$, peso entre 400 e $587 \mathrm{~g}$ e a idade variou entre seis e 14 meses. Somente na análise do raspado de muco corporal foram detectados parasitos, sendo identificados, exclusivamente, espécimes do gênero Trichodina em 34 dos 120 peixes analisados, em duas coletas, resultando em uma prevalência de $28,33 \%$. No total, 144 espécimes de tricodíneos foram observados e a intensidade média foi de 4,24 parasitos por peixe analisado.

O gênero Trichodina (Fig. 1) possui capacidade de se adaptar a diversos ambientes (Adly et al., 2015), podendo acometer peixes em qualquer idade, porém, sua maior incidência ocorre em juvenis. Comumente não causa problemas em peixes adultos. Seu local de preferência é a pele que pode servir de substrato, ou em casos de parasitismo, estes se alimentam de tecidos, destruindo-os e desenvolvendo problemas nos peixes afetados. A transmissão ocorre pelo contato com a água (Adly et al., 2015).

Na Colômbia, Salas-Benavides et al. (2014) registraram uma prevalência de $21,67 \%$ de Trichodina spp. em 194 exemplares de O. mykkis, nas brânquias e na pele dos peixes criados em tanques-rede, com maior incidência na fase larval, afetando peso e tamanho dos peixes parasitados. Schisler et al. (1999), estudando O. mykkis de vida livre no rio Colorado (Estados Unidos), identificaram diferentes espécies de Trichodina parasitando 112 peixes. $\mathrm{O}$ estudo foi realizado em duas etapas, em anos diferentes, e, mesmo assim, foram encontrados protozoários tricodíneos nos dois anos em que o estudo foi realizado. $\mathrm{Na}$ Turquia, Özer et al. (2010) descreveram prevalência 12,2\% em 206 trutas de sistemas de cultivo, com a infecção restrita à pele e às brânquias.

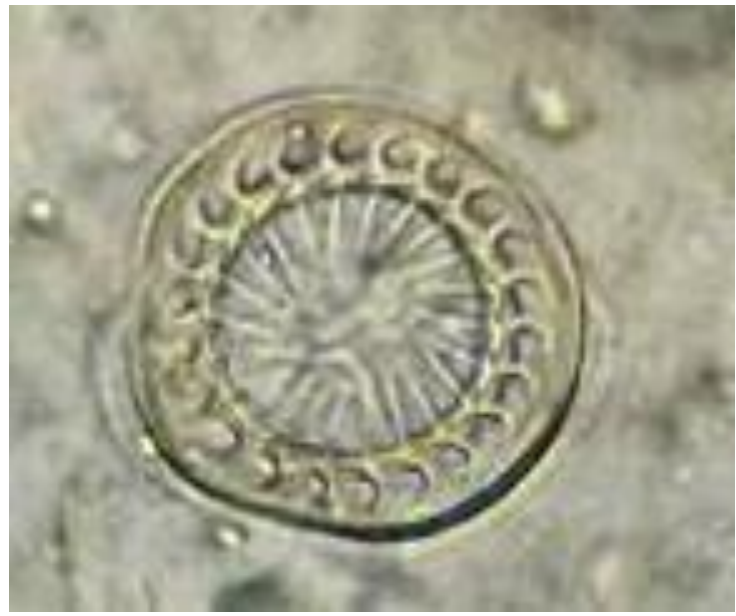

Figura 1. Espécime de Trichodina spp., presente em muco corporal de Oncorhynchus mykiss de criatório comercial em Lages, Santa Catarina. Microscopia óptica (100x).

Fonte: Elaborado pela autora, 2018.

Dentre os estudos realizados no Brasil, Maciel et al. (2018) descreveram ampla distribuição e taxas de ocorrência/prevalência de parasitismo por Trichodina spp. em diferentes espécies de peixes de interesse comercial, tanto silvestres, como de sistemas de criação, porém não relatam a ocorrência em trutas. Trichodina colisae foi identificada em estudos com pacu (Piaractus mesopotamicus) e patinga (híbrido de $P$. mesopotamicus $\times P$. brachypomus) cultivados, respectivamente, no Centro-Oeste e Sudeste do Brasil (Jerônimo et al., 2012b).
Em peixes ornamentais de água doce, no Brasil, foi observado que $51,57 \%$ dos animais estavam parasitados por Trichodina spp., com os "kinguios" (Carassius auratus) apresentando a maior carga parasitária entre as espécies avaliadas (Martins et al., 2012). Martins; Ghiraldelli (2008) relataram a ocorrência de Trichodina magna em 24,7\% (36/146) em tilápias do Nilo (Oreochromis niloticus) coletadas em viveiros de três regiões de Santa Catarina. Hashimoto et al. (2016) descreveram uma nova espécie de Trichodina, $T$. quelenii sp., identificada parasitando Rhamdia quelen e Gymnotus spp. de vida livre e de sistemas 
de criação extensiva, no sul do Brasil. Não foi observada correlação entre os parâmetros físicoquímicos de qualidade da água com os parasitos encontrados (Tab. 1). Os resultados indicam, no presente trabalho, um ambiente adequado para o cultivo de $O$. mykkis.

Tabela 1. Relação entre os parâmetros físico-químicos e os parasitos encontrados, em Oncorhynchus mykiss, Lages, Santa Catarina, 2019

\begin{tabular}{llllll} 
& \multicolumn{1}{c}{$\mathrm{pH}$} & \multicolumn{1}{c}{ Oxigênio dissolvido } & \multicolumn{1}{c}{ Turbidez } & \multicolumn{1}{c}{ Temperatura } & \multicolumn{1}{c}{ Amônia } \\
\hline $\mathrm{r}($ Pearson $)=$ & 0,2375 & $-0,4697$ & $-0,3051$ & 0,4683 & $-0,2328$ \\
IC $99 \%=$ & $-0,85$ a 0,94 & $-0,96$ a 0,75 & $-0,95$ a 0,83 & $-0,75$ a 0,96 & $-0,94$ a 0,85 \\
$(\mathrm{p})=$ & 0,6504 & 0,3472 & 0,5565 & 0,3488 & 0,6571 \\
\hline
\end{tabular}

*r (Pearson): correlação pelo coeficiente de Pearson, IC 99\%: intervalo de confiança, (p): probabilidade de significância.

Fonte: Elaborado pela autora, 2019.

Este é o primeiro relato da ocorrência de Trichodina spp. em trutas-arco-íris criadas em sistema intensivo no Brasil, com baixos índices de infecção parasitária, a qual parecem não ser afetada pelas variáveis físicas e químicas da água.

Palavras-chave: Oncorhynchus mykiss, prevalência, intensidade média, Trichodina

\begin{abstract}
With the aim to determine the prevalence and mean parasite intensity in Oncorhynchus mykiss, 120 specimens were analyzed between April and September 2018. The cavity analysis was done by visual inspection, scraping of mucus, and extraction of the branchial arches. In the evisceration process, the intestine, the liver, and the kidney were separated, while the musculature was analyzed using the "candling table" method. All the collected material was preserved in $10 \%$ buffered formaldehyde and sent to the Laboratory of Parasitology and Parasitic Diseases, of the Center for Agroveterinary Sciences (CAV) of the University of the State of Santa Catarina (UDESC) for making the slides and identifying the parasites. Parasites were detected only in the analysis of the scraping of body mucus. Only specimens of the genus Trichodina were identified, in 34 of the 120 fish analyzed, in two collections, resulting in a prevalence of $28.33 \%$. In total, 144 specimens of Trichodina were observed. The overall mean intensity was of 4.24 parasites in each fish analyzed. Characteristic lesions of infection by protozoa were not identified. This is the first report of the occurrence of Trichodina spp. in O. mykiss bred in an intensive system in Brazil, with low rates of parasitic infection in the mountain region of Santa Catarina.
\end{abstract}

Keywords: Oncorhynchus mykiss, prevalence, mean intensity, Trichodina

\section{REFERÊNCIAS}

ADLY, M.A.; EL-GALIL, M.A.A.A.; SOLIMAN, F.M.; AHMED, F.E.Z.A.A. Histopathological studies on trichodinosis of farmed Oreochomis niloticus. Am. J. Life Sci., v.3, Supl.1, p.30-37, 2015.

BIOESTAT 5.0. Instituto de Desenvolvimento Sustentável Mamirauá. Disponível em: https://www.mamiraua.org.br/ptbr/downloads/programas/. Acesso em: 23 out. 2018.
BUSH, A.O.; LAFFERTY, K.D.; LOTZ, J.M.; SHOSTAK, A.W. Parasitology meets ecology on its own terms: Margolis et al. revisited. J. Parasitol., v.83, p.575-583, 1997.

FOOD and agriculture organization on the United Nations: Oncorhynchus mykiss (Walbaum, 1792). Rome: FAO, [2019]. Available in: http://www.fao.org/fishery/culturedspecies/Onco rhynchus_mykiss/e. Accessed in: 9 May 2019. 
HASHIMOTO, G.S.; MARCHIORI, N.C.; PÁDUA, S.B. et al. A new species of Trichodina Ehrenberg, 1830 (Ciliophora: Trichodinidae) from Rhamdia quelen (Siluriformes: Heptapteridae) and Gymnotus sp. (Teleostei: Gymnotidae) in Brazil. Acta Parasitol., v.61, p.707-712, 2016.

JERONIMO, G.T.; MARCHIORI, N.C.; PÁDUA, S.B. et al. Trichodina colisae (Ciliophora: Trichodinidae): new parasite records for two freshwater fish species farmed in Brazil. Rev. Bras. Parasitol. Vet., v.21, p.366-371, 2012b.

JERÔNIMO， G.T.; TAVARES-DIAS， M.; MARTINS, M.L.; ISHIKAWA, M.M. Coletas de parasitos em peixes de cultivo. Brasília, DF: Embrapa, 2012a. 38p.

MACIEL, P.O.; GARCIA, F.; CHAGAS, E.C. et al. Trichodinidae in commercial fish in South America reviews. Rev. Fish Biol. Fish., v.28, p.33-56, 2018.

MARENGONI, N.G.; SANTOS, R.S.; GONÇALVES JÚNIOR, A.C. et al. Monogenoidea (Dactylogyridae) em tilápias-donilo cultivadas sob diferentes densidades de estocagem em tanques-rede. Arq. Bras. Med. Vet. Zootec., v.61, p.393-400, 2009.
MARTINS, M.L.; GHIRALDELLI, L. Trichodina magna (Van As and Basson, 1989) (Ciliophora: Peritrichia) from cultured Nile tilapia in the state of Santa Catarina, Brazil. Braz. J. Biol., v.68, p.169-172, 2008.

MARTINS, M.L.; MARCHIORI, N.; ROUMBEDAKIS, K.; LAMI, F. Trichodina nobilis (Chen, 1963) and Trichodina reticulata (Hirschmann et Partsch, 1955) from ornamental freshwater fishes in Brazil. Braz. J. Biol., v.72, p.282-286, 2012.

ÖZER, S.; KOYUNCU, E.; DÖNMEZ, E.; SEVIM BULDUKLU, P. Protozoan ectoparasites of Rainbow Trout (Oncorhynchus mykiss, Walbaum, 1792) cultivated in Mersin. Pendik Vet. Mikrobiyol. Derg., v.37, 2010.

SALAS-BENAVIDES, J.; LÓPEZ-MACÍAS, J.N.; ORTEGA-SALAS， A.L.; GÓMEZNIEVES, V.Y. Caracterización parasitaria de la trucha arco-íris (Oncorhynchus mykiss) y su efecto en la producción de la estación piscícola flotante Intiyaco, en el lago Guamuez (Nariño). Vet. Zootec., v.8, p.87-101, 2014.

SCHISLER, G.J.; WALKER, P.G.; CHITTUM, L.A.; BERGERSEN, E.P. Gill ectoparasites of juvenile rainbow trout and brown trout in the upper Colorado river. J. Aquat. Anim. Health, v.11, p.170-174, 1999. 